\title{
BOND GRAPH ANALYSIS IN ROBUST ENGINEERING DESIGN
}

\author{
M. A. ATHERTON ${ }^{1 *}$ AND R. A. BATES ${ }^{2}$ \\ ${ }^{1}$ School of Engineering Systems \& Design, South Bank University, London SE1 OAA, UK \\ ${ }^{2}$ Department of Statistics, University of Warwick, Coventry CV4 7AL, UK
}

\begin{abstract}
SUMMARY
Within engineering design, optimization often involves building models of working systems to improve design objectives such as performance, reliability and cost. Bond graph models express systems in terms of energy fow and can be used to identify key factors that inf uence system behaviour. Robust Engineering Design (RED) is a strategy for the optimization of systems through experimentation and empirical modelling; however, experiments can often be prohibitively expensive for large or complex systems. By using bond graphs as a front-end to RED, experiments on systems could be designed more eff ciently, reducing the number of experiments required for accurate empirical modelling. Two case study examples are given which show that bond graphs can be used to good effect in the empirical analysis of engineering systems. Copyright $\odot 2000$ John Wiley \& Sons, Ltd.
\end{abstract}

KEY WORDS: bond graphs; experimental design; spatial modelling; optimization; robust engineering design

\section{INTRODUCTION}

Engineering systems are modelled so that the relationships between the design variables (design factors) and the desired system response (responses) may be understood and controlled. Accurate models allow systems to be optimized with respect to design objectives such as performance, reliability and cost. The level of accuracy obtained by system models depends on the level of understanding of the system, the cost of building the model and the cost of subsequent model evaluation. In practice, system models approximate system behaviour by appropriate linearizations and this needs to be taken into account during analysis.

In the $f$ eld of engineering design complex engineering systems are routinely modelled for the purpose of design optimization. This is not often a straightforward process, engineering systems often exist in several states, models of the system may be very large and costly to evaluate, there can be multiple responses to optimize and these responses are often subject to multiple constraints. Robust Engineering Design (RED) is a strategy for experimentation, modelling and optimization of engineering systems borne out of the mathematical disciplines of Design of Experi-

\footnotetext{
*Correspondence to: M. Atherton, School of Engineering Systems \& Design, South Bank University, Southwark Campus, 103 Borough Road, London SE1 0AA, UK.
}

ments (DoE), Response Surface Modelling (RSM) and numerical optimization. Accurate modelling of large systems can require a large number of observations, especially when the number of factors is large and the responses are nonlinear. Conducting experiments eff ciently requires the gathering of as much prior information about the system as possible. The design factors that are to be the subject of experimentation need to be identifie , including which factors are likely to be the most important, the existence of complex relationships between factors, the practical ranges of factor values and effective ways of measuring system response.

Bond graph models of systems express relationships between design factors using energy fow and are built using engineering insight into the physical nature of a system. Building a bond graph model of a system can highlight key design factors and their relationships and encapsulate the engineering knowledge that has led to the initial system architecture and factor settings.

The aim of this paper is to explore a new strategy for performing RED in a complex engineering environment, capturing engineering knowledge through the use of bond graphs and using this as a frontend for RED. Two case studies are presented, the design of a loudspeaker driver unit and the design of a hedgetrimmer. Bond graph models are built for each design and used to collect empirical data. In the loudspeaker study, data were collected 'on-line' during the 


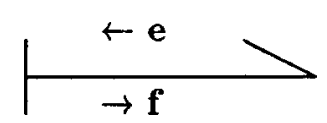

Figure 1. Power bond with causal stroke

production of 20 driver units. In the hedgetrimmer study, a planned experiment was conducted on the design, and 'off-line' data were collected for analysis.

\section{BOND GRAPHS}

Bond graphs are a means of generating rapid mathematical models of multi-energy domain systems that were introduced by Paynter [1] based on electromechanical analogues. Notable contributions have been made by Karnopp [2], Rosenberg [3] and Cellier [4]. The causality assignment and modelbuilding associated with bond graphs makes them an interesting proposition for use as a RED front-end.

Dynamic physical systems are concerned with one or more of the following: (i) energy transfer, (ii) mass transfer, and (iii) information (or signal) transfer. Bond graphs are an abstract representation of a system that uses one set of symbols to represent all applicable types of systems in terms of energy transfer [5]. In particular, they focus on the exchange of power between components.

Each line or bond with half arrow (Figure 1) in a bond graph implies the existence of a pair of signals whose fows are in opposite directions. These signal pairs, or power variables, are generally termed effort (e) and fow (f). As most engineering systems are dynamic these power variables are often functions of time.

Only a few basic types of element are required in order to model a variety of energy domains $[6,7]$. The elements will have one or more ports and at each port, effort and fow variables co-exist. In addition, one of these variables will be controlled, but both cannot be controlled simultaneously. The direction of the half arrowhead on the bond in Figure 1 indicates the direction of positive power fow [6]. The short vertical bar, or causal stroke, indicates how e and $\mathrm{f}$ are simultaneously determined on a bond, i.e. effort pushes towards the causal stroke and fow fows away from it.

The study of input-output causality is a unique feature of bond graphs and can indicate the form of the underlying mathematical representation of the system. This can be very useful in avoiding analytical problems, such as unnecessary differential calculus [7]. In mathematical modelling the organization of component constitutive laws into sets of differential equations requires cause-and-effect decisions to be made [8]. The integral causality assignment of bond graphs aids in the development of mathematical models that mimic the physical system they represent and that can be resolved avoiding unnecessary complexity [6].

\section{ROBUST ENGINEERING DESIGN}

RED is concerned with reducing the effects of uncontrollable factors (noise factors) on the output of a system by selecting optimum settings for controllable factors (design factors) through the use of Experimental Design [9]. In terms of factor selection, RED involves the following steps:

(i) identify high-level factors, such as overall functions, quality characteristics or physical effects;

(ii) use physical laws to identify the role of lowlevel factors, that is to highlight signif cant design factors;

(iii) estimate appropriate target values for (lowlevel) design factors in order to achieve optimal performance;

(iv) identify the values to be employed for these design factors as they will depend upon production capability and other statistical issues.

The 'engineering judgement' exercised in step (ii) and step (iii) above often appears to be based on insight into the nominal behaviour of the system under investigation gained through analysis and/or experience of similar systems. Effective insight would ideally provide understanding of the inf uences on system output variability as well as nominal output, but this is rarely the case. For example, physical laws could be used to reveal complex relationships between high-level and low-level factors in order to assess potential variability. However, insight is often limited to simple relationships for the purpose of identifying target values for (low-level) design factors and potential interactions between them are overlooked.

Dealing with interactions between design factors often requires compromise and trade-off between the target design factor values in question (step (iii) above). Consequently, the selection of design factors and the anticipation of relationships between them are an unreliable aspect of contemporary RED practice making the predictive power of the method unreliable. Unexpected interactions discovered later in the design process may require design changes in order to 
reduce their effects. There is also the risk that key design factors will not be identif ed for inclusion in the experiments because of insuff cient understanding of the system. Thus the conventional approach to RED relies heavily on physical experiments that can be time-consuming and costly. Energy transfer has been highlighted as a key consideration of many physical systems when selecting parameters for an RED experiment [10], and building an energy-based model of the system could aid the design factor selection process.

To identify key design and noise factors prior to physical experimentation requires highly complex computer models capable of simulating variation (noise), which is not yet practical with current analysis tools. It is well known from reliability engineering that representative probability distributions of loads are virtually impossible to identify [11], which means that physical experiments should remain a stage in RED for the foreseeable future. Instead, the focus is on using appropriate computer models to provide insight into physical experiments. For example, subsystem identif cation for grouping of variables is especially useful in reducing system complexity.

\section{BOND GRAPHS AS AN RED FRONT-END}

Since equations do not normally express energy fow within a system this cannot be observed analytically. However, engineers are often interested in developing a 'feel' for energy fow in physical systems. One advantage of the bond graph representation is that the system topology is maintained, giving an idea of the causal relationships between parameters, which, in turn, offers some guidance on parameter selection. Such an insight into system behaviour is important when planning RED experiments on energy-related products so that appropriate design and noise factors are included. The use of bond graphs is proposed in order to highlight the role of energy-based parameters in RED.

Another useful property of bond graphs is that any design factors represented are all at the same 'level' of complexity or detail within the system. The link between the bond graph graphical representation and the computational causality was clearly demonstrated in an air pump example [12]. It is commonly accepted $[3,4]$ that with suff cient practice, identificatio of potential significan system parameters can be made solely from the bond graph representation. This has not been demonstrated for RED and so the following procedure is suggested.

(i) Draw the bond graph of the system ensuring

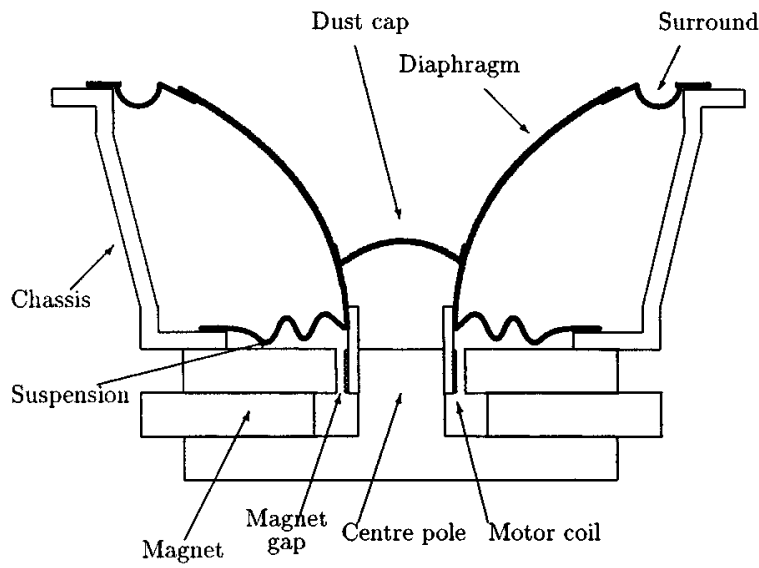

Figure 2. Loudspeaker driver unit

integral causality (following the Sequential Causality Assignment Procedure [6]).

(ii) Obtain a feel for the significan design factor through visualizing or sketching the statespace equations and assigning estimated values, including a sensitivity analysis on these values.

(iii) Select each inertia and capacitance from amongst the chosen design and trace the causal links to highlight potential interactions between design factors.

(iv) Use this information to select factors and responses for RED.

Two case studies are now presented. Their main aim is to show how the bond graph method can be used to describe engineering systems and provide useful information for an empirical analysis. The fir $\mathrm{t}$ case study is the design of a loudspeaker driver unit and involves building a bond graph of the system and collecting data on the manufacture of 20 units. The results of the bond graph analysis are compared with the relative importance of the design factors as predicted by the empirical data collected during production. The second study is the design of a hedgetrimmer. Here the bond graph model is used to plan a small experiment to determine the relative effects of the design factors.

\section{LOUDSPEAKER VOICE COIL CASE STUDY}

\subsection{Driver unit parameters}

Loudspeaker performance will vary between any two speakers taken from the production line due to the inevitable variation in material properties, dimensions, and other parameters of the component parts. The two major subsystems of a loudspeaker are the driver unit 


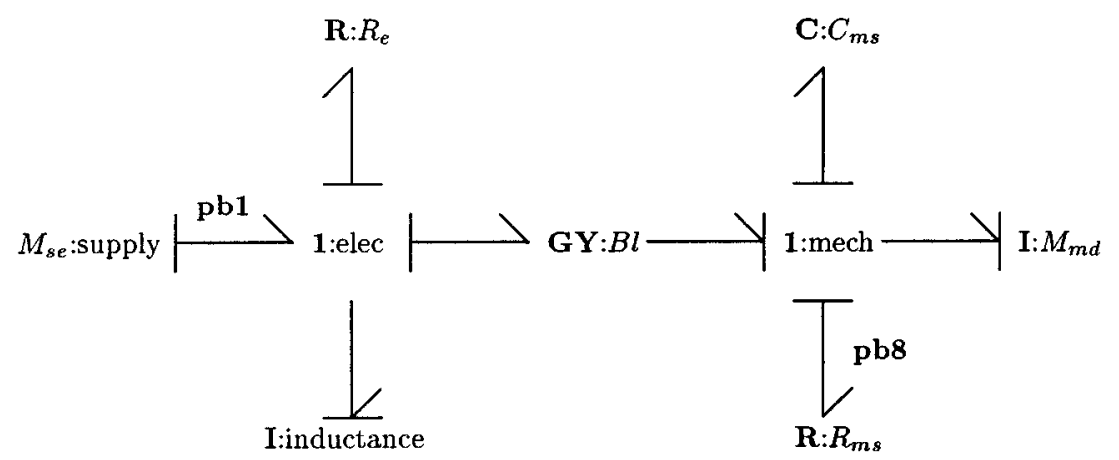

Figure 3. Bond graph model of loudspeaker voice-coil

and its enclosure. The main aim of this case study is to investigate the main sources of unit-to-unit variation of driver units.

The basic working principle of the moving-coil loudspeaker can be appreciated from the driver unit assembly shown in Figure 2. Essentially a motor coil moves axially within a radial magnetic fiel driving a diaphragm at audible frequencies radiating sound from its surface into the air. Consideration of potential design factors in the driver unit for RED experimentation highlights several groups or subsystems even for this product with its relatively low parts count. The loudspeaker design factors will depend on the nature of the subsystem to which they are associated.

(i) Surround - material and adhesive bonding properties

(ii) Diaphragm - material properties and various dimensions

(iii) Suspension-dynamic characteristics

(iv) Magnet-magnetic properties and various dimensions

(v) Voice-coil-energy properties and various dimensions

Choosing factors for experimentation from one system level is preferred in order to avoid interacting effects [9]. From this basic level (low resolution) there is potential for more detail to be added by subdividing the system into more elements and connections. The next Section follows steps (i) to (iii) of Section 4 to construct the bond graph model.

\subsection{Bond graph model of voice-coil}

Figure 3 was utilized to highlight potential design factors for selection from a large number of parameters identif ed by the design team. This bond graph is a lumped parameter model. Distributed parameter models are a more advanced bond graph approach, but would involve considerable time to model, and such models have not yet been presented in the bond graph literature [13].

In choosing the design factors for this investigation, the bond graph in Figure 3 highlights parameters at a common basic level linked with the fow of power through the voice-coil device. That is for each energy domain (electrical and mechanical in this case) the various parameters are grouped or 'lumped' and assigned to categories for 1-port elements if they store or dissipate energy, and a 2-port element if they convert energy, namely:

1. $R_{e}$ - the electrical resistance of the driver unit, made up almost entirely by that of the coil with a small amount contributed by the lead-out braid;

2. $B l$-the motor 'shove factor', determined by the coil turns on the voice coil and the magnetic fl $\mathrm{x}$ generated in the gap between magnet and coil;

3. $M_{m d}$ - the total moving mass, which is mainly that of the voice coil and the cone diaphragm;

4. $R_{m s}$ - the total mechanical resistance offered from elements such as the surround and support; and

5. $C_{m s}$ - the total mechanical compliance of the supports.

Element (2) is a 2-port element, the rest are 1-port elements. The coil inductance, I, was not selected under guidance from the engineering design team.

It is important to note that this bond graph model is an approximation to the loudspeaker system, but that the bond graph approach does allow modelling of nonlinear behaviour. Some nonlinear effects, such as non-uniformity of the magnetic feld at the edge of the loudspeaker magnet, have been ignored, but these effects can be reduced by careful design, for example, flu linearity can be improved by using devices such as undercuts in the magnet poles and eddy current control rings [14]. 


\subsection{State equations for design factor selection}

The state equations are determined from the Power Bonds in Figure 3 and numbered clockwise around each junction starting with the supply as Power Bond 1 (marked as pb1 in Figure 3), which has associated effort $e_{1}$ and fow $f_{1}$, and fi ishing with the $R_{m s}$ as Power Bond 8 (marked as pb8 in Figure 3).

The bond graph equations can be written as follows:

$$
\begin{aligned}
\frac{\mathrm{d} f_{4}}{\mathrm{~d} t} & =\frac{1}{I}\left(M_{s e}-R_{e} f_{4}-B l f_{7}\right) \\
\frac{\mathrm{d} e_{6}}{\mathrm{~d} t} & =\frac{1}{C_{m s}} f_{7} \\
\frac{\mathrm{d} f_{7}}{\mathrm{~d} t} & =\frac{1}{M_{m d}}\left(B l f_{4}-e_{6}-R_{m s} f_{7}\right)
\end{aligned}
$$

Putting these equations in matrix form:

$$
\begin{aligned}
{\left[\begin{array}{c}
\frac{\mathrm{d} f_{4}}{\mathrm{~d} t} \\
\frac{\mathrm{d} e_{6}}{\mathrm{~d} t} \\
\frac{\mathrm{d} f_{7}}{\mathrm{~d} t}
\end{array}\right]=} & {\left[\begin{array}{ccc}
\frac{R_{e}}{I} & 0 & -\frac{B l}{I} \\
0 & \frac{1}{C_{m s}} & 0 \\
\frac{B l}{M_{m d}} & \frac{1}{M_{m d}} & -\frac{R_{m s}}{M_{m d}}
\end{array}\right]\left[\begin{array}{l}
f_{4} \\
e_{6} \\
f_{7}
\end{array}\right] } \\
& +\left[\begin{array}{c}
\frac{1}{I} \\
0 \\
0
\end{array}\right]\left[M_{s e}\right]
\end{aligned}
$$

This reduces to:

$$
\begin{aligned}
{\left[\begin{array}{c}
\frac{\mathrm{d} f_{4}}{\mathrm{~d} t} \\
\frac{\mathrm{d} e_{6}}{\mathrm{~d} t} \\
\frac{\mathrm{d} f_{7}}{\mathrm{~d} t}
\end{array}\right]=} & {\left[\begin{array}{ccc}
2 \times 10^{4} & 0 & -2.4 \times 10^{4} \\
0 & 1 \times 10^{3} & 0 \\
750 & 125 & -50
\end{array}\right]\left[\begin{array}{c}
f_{4} \\
e_{6} \\
f_{7}
\end{array}\right] } \\
& +\left[\begin{array}{c}
4 \times 10^{3} \\
0 \\
0
\end{array}\right]\left[M_{s e}\right]
\end{aligned}
$$

The relative significanc of the design factors in the main matrix can be estimated after scaling the matrix so as to equalize all numerical values [12]. First estimates of the nominal values of $f_{4}, e_{6}$ and $f_{7}$ are required. Let us consider $f_{4}=3 \mathrm{~A}, e_{6}=1 \mathrm{~N}$ and $f_{7}=1 \mathrm{~m} \mathrm{~s}^{-1}$. Scaling $e_{6}$ and $f_{7}$ by 3 yields the following equations:

$$
\begin{aligned}
{\left[\begin{array}{c}
\frac{\mathrm{d} f_{4}}{\mathrm{~d} t} \\
\frac{\mathrm{d} 3 e_{6}}{\mathrm{~d} t} \\
\frac{\mathrm{d} 3 f_{7}}{\mathrm{~d} t}
\end{array}\right]=} & {\left[\begin{array}{ccc}
2 \times 10^{4} & 0 & -8 \times 10^{3} \\
0 & 3 \times 10^{3} & 0 \\
2.25 \times 10^{3} & 125 & -50
\end{array}\right] } \\
& \times\left[\begin{array}{c}
f_{4} \\
3 e_{6} \\
3 f_{7}
\end{array}\right]+\left[\begin{array}{c}
4 \times 10^{3} \\
0 \\
0
\end{array}\right]\left[M_{s e}\right]
\end{aligned}
$$

The two largest values indicate that from the design factors considered, $R_{e}$ and $B l$ may have signif cant inf uence on the energy fow through the voice-coil. Thus we expect these two factors to be particularly signif cant in physical experiments.

\subsection{Driver unit test data}

The 20 voice coils remaining were assembled into driver units using parts specificall selected from the production line for their near-nominal values. That is, apart from the variation in voice coil measurements, the driver units were considered to be 'best practice' in terms of manufacture.

It is diff cult to measure $M_{m d}, R_{m s}$ and $C_{m s}$ dynamically in a direct manner. To obtain measurements for the above design factors, the following three dimensionless parameters, of specif c interest to the loudspeaker design engineers, are estimated using a Fast Fourier Transform analyser with $100 \mathrm{~Hz}$ bandwidth pseudo-random noise:

1. $Q_{e s}$ - the electrical damping ratio define as $\left(2 \pi f_{s} M_{m d} R_{e}\right) /(B l)^{2}$;

2. $Q_{m s}$ - the mechanical damping ratio define as $\left(2 \pi f_{s} M_{m d}\right) / R_{m s}$;

3. $Q_{t s}$ - the total system damping ratio def ned as $1 /\left(\left(1 / Q_{m s}\right)+\left(1 / Q_{e s}\right)\right)$

where $f_{s}$ is the free resonance frequency.

The procedure for determining parameter values is as follows.

(i) Measure fundamental resonance $f_{s}$ and $Q_{m s}$. This gives expressions involving $M_{m d}, C_{m s}, R_{m s}$ and $B l$.

(ii) Add a fixe mass to the driver cone and re-measure $f_{s} . M_{m d}$ and $C_{m s}$ can now be evaluated with reasonable accuracy (from $f_{0}=$ $(1 / 2 \pi) \sqrt{k / m})$.

iii. Measure real part of impedance at $f_{s}$. This gives $R_{e s}=(B l)^{2} / R_{m s}$.

(iv) Put values into expression for $Q_{m s}$, i.e. $Q_{m s}=$ $2 \pi f_{s} M_{m d} R_{e s} /(B l)^{2}$. Now we have $B l$ and therefore $R_{m s}$ also.

(v) Measure piston diameter and calculate mass of air load, subtract from $M_{m d}$ to get actual piston mass.

(vi) Calculate $Q_{e s}$ from $Q_{m s}, R_{m s}$ and $R_{e}$. Total $Q$, $Q_{t s}$, is the parallel sum of $Q_{e s}$ and $Q_{m s}$.

One can see already that there is potentially a large inaccuracy in the measurement of $B l$ since it is a derived parameter. Also, $Q_{m s}$, although based on measurement, can also be subject to errors as it is calculated by measuring loudspeaker electrical impedance, subtracting the resistance and inductive effects and using a simple curve-fitti g routine to determine the $3 \mathrm{~dB}$ points (the loudspeaker can be easily modelled as it is a single degree of freedom system). The measured values for 20 driver units are shown in Table 1. 
Table 1. Driver-unit parameter values

\begin{tabular}{cccccccccc}
\hline $\begin{array}{c}\text { Driver } \\
\text { unit }\end{array}$ & $\begin{array}{c}R_{e} \\
(\Omega)\end{array}$ & $\begin{array}{c}f_{s} \\
(\mathrm{~Hz})\end{array}$ & $Q_{m s}$ & $Q_{t s}$ & $Q_{e s}$ & $\begin{array}{c}M_{m d} \\
(\mathrm{~g})\end{array}$ & $\begin{array}{c}C_{m s} \\
\left(10^{-3} \mathrm{~m} \mathrm{~N}^{-1}\right)\end{array}$ & $\begin{array}{c}B l \\
(\mathrm{~T} \mathrm{~m})\end{array}$ & $\begin{array}{c}R_{m s} \\
\left(\mathrm{~kg} \mathrm{~s}^{-1}\right)\end{array}$ \\
\hline 1 & 4.67 & 54.46 & 7.45 & 0.363 & 0.381 & 7.835 & 1.015 & 5.942 & 0.387 \\
2 & 4.75 & 49.63 & 7.15 & 0.322 & 0.337 & 8.392 & 1.142 & 6.280 & 0.392 \\
3 & 4.94 & 54.43 & 7.39 & 0.368 & 0.388 & 7.882 & 1.012 & 6.067 & 0.390 \\
4 & 4.86 & 54.48 & 7.43 & 0.386 & 0.408 & 7.855 & 1.012 & 5.862 & 0.387 \\
5 & 4.85 & 54.69 & 7.39 & 0.361 & 0.380 & 8.042 & 0.982 & 6.145 & 0.400 \\
6 & 4.87 & 53.35 & 7.69 & 0.365 & 0.383 & 8.120 & 1.022 & 6.080 & 0.380 \\
7 & 4.86 & 53.35 & 7.50 & 0.351 & 0.368 & 8.125 & 1.022 & 6.202 & 0.387 \\
8 & 4.76 & 53.37 & 8.47 & 0.352 & 0.367 & 8.167 & 1.020 & 6.162 & 0.342 \\
9 & 4.92 & 53.88 & 7.20 & 0.362 & 0.381 & 7.967 & 1.022 & 6.105 & 0.402 \\
10 & 4.83 & 53.74 & 7.84 & 0.349 & 0.365 & 8.005 & 1.025 & 6.192 & 0.370 \\
11 & 4.85 & 53.09 & 7.17 & 0.364 & 0.385 & 8.315 & 1.017 & 6.107 & 0.412 \\
12 & 4.92 & 52.22 & 7.59 & 0.342 & 0.359 & 8.302 & 1.047 & 6.317 & 0.382 \\
13 & 4.74 & 54.20 & 8.09 & 0.363 & 0.380 & 7.577 & 1.062 & 5.882 & 0.342 \\
14 & 4.97 & 52.18 & 7.74 & 0.356 & 0.373 & 8.037 & 1.080 & 6.130 & 0.367 \\
15 & 5.00 & 52.38 & 7.53 & 0.355 & 0.373 & 8.135 & 1.062 & 6.195 & 0.382 \\
16 & 4.76 & 48.91 & 7.41 & 0.312 & 0.326 & 8.092 & 1.222 & 6.240 & 0.357 \\
17 & 4.92 & 54.68 & 7.89 & 0.368 & 0.386 & 8.197 & 0.965 & 6.192 & 0.380 \\
18 & 4.86 & 52.88 & 7.41 & 0.350 & 0.367 & 8.242 & 1.030 & 6.222 & 0.392 \\
19 & 4.87 & 49.88 & 6.85 & 0.320 & 0.336 & 8.262 & 1.152 & 6.330 & 0.402 \\
20 & 4.79 & 51.48 & 7.74 & 0.345 & 0.361 & 8.497 & 1.057 & 6.243 & 0.380 \\
\hline
\end{tabular}

\subsection{Sound Pressure Level measurement}

The Sound Pressure Level (SPL) of each driver unit was measured in a inf nite baff e anechoic chamber, the driver units being tested in a random order. The results are shown in Figure 4, where SPL is plotted in the frequency range of 100 to $800 \mathrm{~Hz}$.

\subsection{Linear regression}

In the analysis two replications were used for each coil in each of which a fully frequency response curve was produced. The explanatory factors modelled are:

(i) coil electrical resistance including connections $\left(R_{e}\right)$

(ii) total moving mass $\left(M_{m d}\right)$;

(iii) total suspension compliance $\left(C_{m s}\right)$;

(iv) motor 'shove factor' $(B l)$;

(v) mechanical damping $\left(R_{m s}\right)$.

A set of simple linear regression analysis of the experimental data is reported in Table 2, where the factors are modelled at 10 specif $\mathrm{c}$ frequency points on the SPL curves. The factor values are scaled to be in the range $[-0.5,+0.5]$ so that direct comparisons may be made between model factors.

The regression results show that the ftted linear models account for between 10 and $64 \%$ of the variability in the data depending on which response was chosen. The regression models between 100 and $400 \mathrm{~Hz}$ on average explain about $60 \%$ of the variation in the data, with an $F$ statistic of around 4 and association $95 \% p$-value of 0.02 . This indicates that it is unlikely that all the regression coefficie ts are zero for these models. From the models of 150 to $400 \mathrm{~Hz}$, the coeff cient for $R_{e}$ has the highest value and is the most significan factor.

\section{HEDGETRIMMER CASE STUDY}

The hedgetrimmer is a simple electromechanical device for cutting small branches and stems. The no-load running speed of each manufactured product is dispersed around the design performance target depending on the actual parameter values achieved for the motor, gearbox and blade subsystems, as shown in Figure 5. During operation, the blade speed of an individual product will vary due to the loading placed on the system by the cutting action.

The bond graph of the hedgetrimmer is shown in Figure 6 and is constructed using the energyrelated parameters of the motor and blade subsystems. The model has been developed incorporating signal fows in order to achieve a representation of the reciprocating motion of the blade and the intermittent 


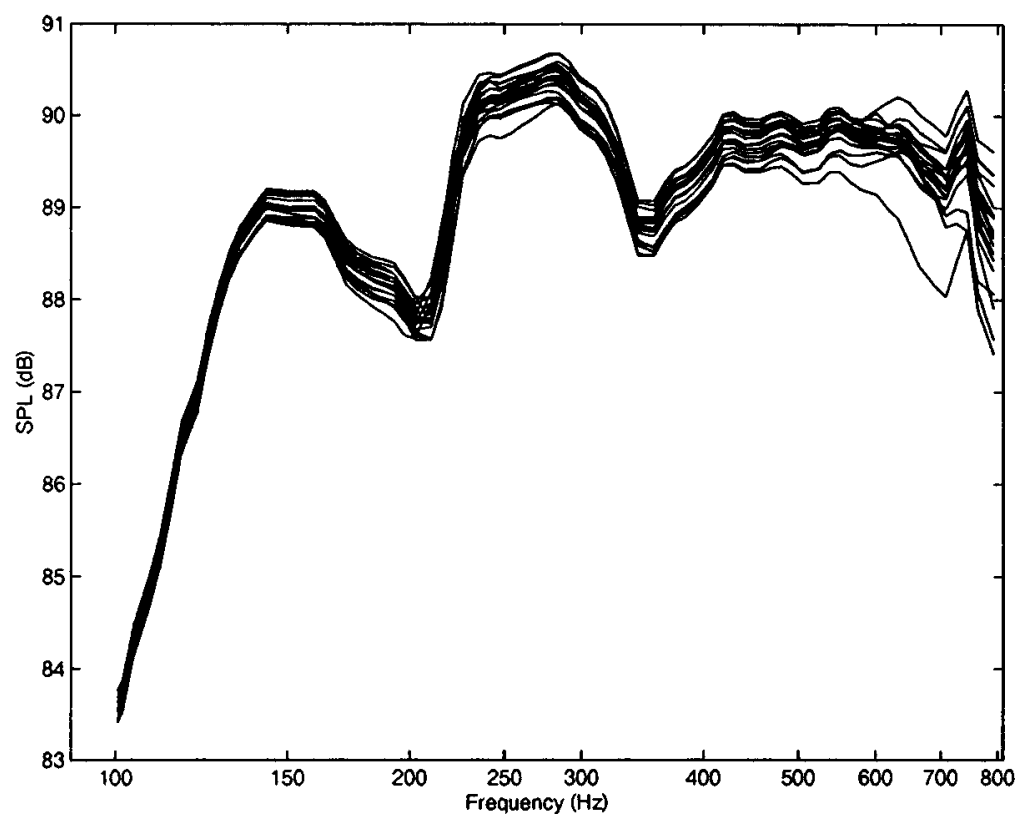

Figure 4. SPL measurements of 20 driver units

Table 2. Linear regression model results - most signif cant factor in bold for each model

\begin{tabular}{|c|c|c|c|c|c|c|c|c|c|}
\hline \multirow{2}{*}{$\begin{array}{l}\text { Freq. } \\
(\mathrm{Hz})\end{array}$} & \multicolumn{6}{|c|}{ Regression model factors } & \multicolumn{3}{|c|}{ Regression statistics } \\
\hline & Constant & $R_{e}$ & $M_{m d}$ & $C_{m s}$ & $B l$ & $R_{m s}$ & $R^{2}$-value & $F$-value & $p$-value \\
\hline 100 & 83.64 & -0.0995 & 0.2244 & 0.1205 & -0.1665 & -0.2045 & 0.5178 & 3.007 & 0.0476 \\
\hline 150 & 89.00 & -0.2891 & 0.0946 & 0.0129 & 0.1086 & -0.1709 & 0.6447 & 5.080 & 0.0073 \\
\hline 200 & 87.91 & -0.2771 & 0.0952 & 0.1468 & 0.1038 & -0.1844 & 0.6223 & 4.614 & 0.0107 \\
\hline 250 & 90.25 & -0.2223 & 0.1447 & 0.2209 & 0.0414 & -0.2016 & 0.4959 & 2.754 & 0.0618 \\
\hline 300 & 90.19 & -0.3485 & 0.0130 & 0.1561 & 0.1175 & -0.1979 & 0.6107 & 4.392 & 0.0129 \\
\hline 400 & 89.40 & -0.3089 & 0.0192 & 0.1357 & 0.1740 & -0.2367 & 0.5819 & 3.897 & 0.0201 \\
\hline 500 & 89.73 & -0.2760 & 0.1525 & 0.0923 & 0.1527 & -0.2088 & 0.5074 & 2.884 & 0.0539 \\
\hline 600 & 89.79 & -0.2351 & 0.1472 & 0.1090 & 0.1558 & -0.1469 & 0.2960 & 1.177 & 0.3684 \\
\hline 700 & 89.35 & -0.2260 & 0.3001 & 0.3133 & -0.2429 & -0.0757 & 0.1009 & 0.3141 & 0.8963 \\
\hline 800 & 88.74 & 0.2434 & -0.7266 & 0.3572 & -0.3155 & 0.7197 & 0.1866 & 0.6422 & 0.6717 \\
\hline
\end{tabular}
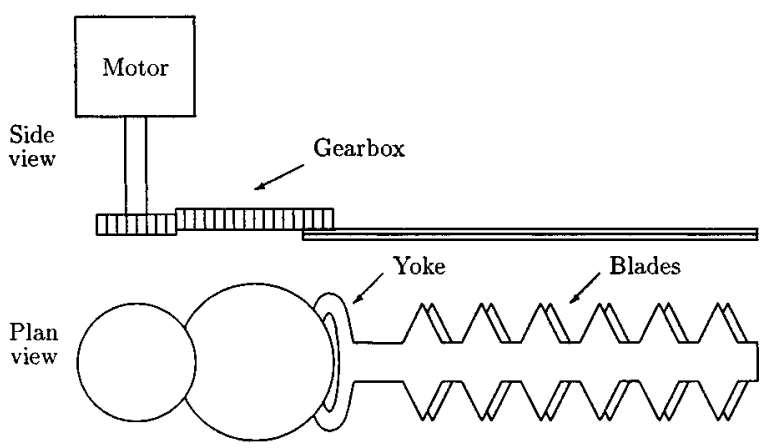

Figure 5. Schematic of hedgetrimmer nature of the load. The load is conf gured to always work against motion, peaking at half-stroke, i.e. out of phase with the blade motion. The operation of this model has been verifie using a commercially available bond graph simulation package, 20Sim, available at: www.20sim.com.

The function $f_{1}(x)$ in Figure 6 represent functions for changing displacement into an intermittent load to simulate the cutting action on branches, and the function $f_{2}(x)$ represents a function to convert rotary displacement into linear displacement. Typical bond graph model factors are shown in Table 3.

The typical values established for each factor were calculated from physical measurements of motors and blades and existing product test data. Note that there 


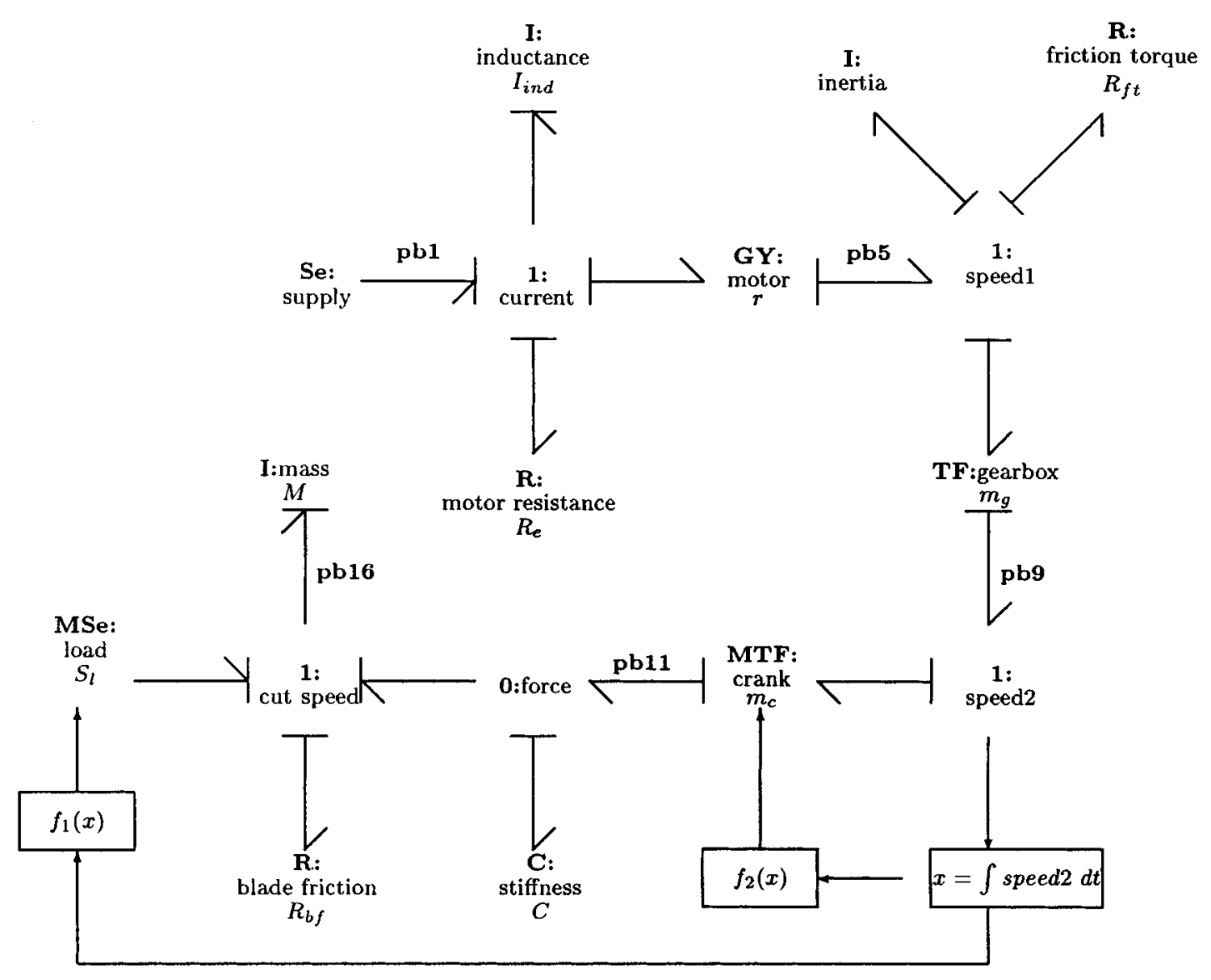

Figure 6. Bond graph model of hedgetrimmer

Table 3. Hedgetrimmer bond graph model factors

\begin{tabular}{|c|c|c|}
\hline & Description & Typical value \\
\hline$R_{e}$ & $\begin{array}{l}\text { Electrical resistance of } \\
\text { motor }\end{array}$ & $30.3 \Omega$ \\
\hline$I_{\text {ind }}$ & Motor inductance & $0.152 \mathrm{H}$ \\
\hline$r$ & $\begin{array}{l}\text { Modulus of motor } \\
\text { gyrator element }\end{array}$ & 0.074 \\
\hline$I$ & $\begin{array}{l}\text { Moment of inertia of } \\
\text { motor armature }\end{array}$ & $4.54 \mathrm{e}-05 \mathrm{~kg} \mathrm{~m}^{2}$ \\
\hline$R_{f t}$ & $\begin{array}{l}\text { Friction torque of motor } \\
\text { mechanism }\end{array}$ & $15.9 \mathrm{e}-06 \mathrm{Nms} \mathrm{rad}^{-1}$ \\
\hline$m_{g}$ & $\begin{array}{l}\text { Modulus of gearbox } \\
\text { transformer }\end{array}$ & 0.0125 \\
\hline$m_{c}$ & Modulus of yoke crank & 0.082 \\
\hline$C$ & $\begin{array}{l}\text { Mechanical compliance } \\
\text { of system }\end{array}$ & $1 \mathrm{e}-05 \mathrm{~m} \mathrm{~N}^{-1}$ \\
\hline$M$ & Expected blade mass & $0.5 \mathrm{~kg}$ \\
\hline$R_{b f}$ & Blade friction & $6.3 \mathrm{Ns} \mathrm{m}^{-1}$ \\
\hline$S_{l}$ & $\begin{array}{l}\text { Cutting load opposing } \\
\text { motion }\end{array}$ & $23 \mathrm{~N}$ and $45 \mathrm{~N}$ \\
\hline
\end{tabular}

are two values associated with the factor $S_{l}$, which represent the distribution of foliage from thin objects such as leaves to thick objects such as branches. The factor $S_{l}$ can be considered as a noise factor, as it is not under the designer's control.

As for the loudspeaker bond graph model, the hedgetrimmer model is an approximation to the real system, but can be used to describe important nonlinear behaviour of the system.

\subsection{Bond graph insight into hedgetrimmer system}

The bond graph of Figure 6 is used to construct a set of state-space equations describing the system, recalling that the state-space equations are generated from consideration of each inertia and capacitor element in the system. From Figure 6, each power bond (pb) considered below is assigned a number clockwise around each 1-junction and 0 -junction starting with the supply as pb1 the gyrator output as pb5, the gearbox output as pb9, the crank output as 
pb11 and f nishes with the mass as pb16. As for the previous bond graph, the variables are numbered so that $e_{1}$ and $f_{1}$ are the effort and fow associated with pb1 and so on.

Thus at the frst 1-junction

$$
\frac{\mathrm{d} f_{2}}{\mathrm{~d} t}=\frac{1}{I_{\text {ind }}}\left(S_{e}-R_{e} f_{2}-r f_{6}\right)
$$

and for the second 1-junction

$$
\frac{\mathrm{d} f_{6}}{\mathrm{~d} t}=\frac{1}{I}\left(r f_{2}-R_{f t} f_{6}-m_{g} m_{c} e_{13}\right) .
$$

For the 0 -junction:

$$
\frac{\mathrm{d} e_{13}}{\mathrm{~d} t}=\frac{1}{C}\left(m_{c} m_{g} f_{6}-f_{16}\right)
$$

and for the f nal 1-junction

$$
\frac{\mathrm{d} f_{16}}{\mathrm{~d} t}=\frac{1}{M}\left(e_{13}-R_{b f} f_{16}+S_{l}\right) .
$$

Putting these equations into matrix form gives

$$
\begin{aligned}
\frac{\mathrm{d}}{\mathrm{d} t}\left[\begin{array}{c}
f_{2} \\
f_{6} \\
e_{13} \\
f_{16}
\end{array}\right]= & {\left[\begin{array}{cccc}
\frac{-R_{e}}{I_{\text {ind }}} & \frac{-r}{I_{\text {ind }}} & 0 & 0 \\
\frac{r}{I} & \frac{-R_{f t}}{I} & \frac{-m_{g} m_{c}}{I} & 0 \\
0 & \frac{m_{c} m_{g}}{C} & 0 & \frac{-1}{C} \\
0 & 0 & \frac{1}{M} & \frac{-R_{b f}}{M}
\end{array}\right]\left[\begin{array}{c}
f_{2} \\
f_{6} \\
e_{13} \\
f_{16}
\end{array}\right] } \\
& +\left[\begin{array}{cc}
\frac{1}{I_{\text {ind }}} & 0 \\
0 & 0 \\
0 & 0 \\
0 & \frac{1}{M}
\end{array}\right]\left[\begin{array}{c}
S_{e} \\
S_{l}
\end{array}\right] .
\end{aligned}
$$

As was shown for the voice-coil, the relative significanc of the design factors in the main matrix can be estimated after scaling the matrix so as to equalize all numerical values [12]. However, estimates of the nominal values of $f_{2}, f_{6}, e_{13}$ and $f_{16}$ are $\mathrm{f}$ rst required.

From motor data corresponding to approximate anticipated blade running sped of $1800 \mathrm{rpm}, f_{2}=$ $1.33 \mathrm{~A}, f_{6}=2847 \mathrm{rad} \mathrm{s}^{-1}$ and $f_{16}=1.896 \mathrm{~m} \mathrm{~s}^{-1}$. However, $e_{13}$ is more diff cult to estimate. Let the sum of output power and power lost to blade friction $=74 \mathrm{~W}+66 \mathrm{~W}=140 \mathrm{~W}$, then, based on power $=$ force $\times$ velocity, force $e_{13}=140 / 1.86=75 \mathrm{~N}$.
We can then scale each equation to unity and the matrix equations become

$$
\begin{aligned}
\frac{\mathrm{d}}{\mathrm{d} t}\left[\begin{array}{c}
\frac{f_{2}}{1.33} \\
\frac{f_{6}}{2847} \\
\frac{e_{13}}{1.86}
\end{array}\right] \\
=\left[\begin{array}{cccc}
-199.3 & -1049 & 0 & 0 \\
0.76 & -0.35 & -0.6 & 0 \\
0 & 3898 & 0 & 2480 \\
0 & 0 & 3061 & -38.2
\end{array}\right] \\
\times\left[\begin{array}{c}
\frac{f_{2}}{1.33} \\
\frac{f_{6}}{2847} \\
\frac{e_{13}}{75} \\
\frac{f_{16}}{1.86}
\end{array}\right]+\left[\begin{array}{cc}
4.96 & 0 \\
0 & 0 \\
0 & 0 \\
0 & 1.08
\end{array}\right]\left[\begin{array}{c}
S_{e} \\
S_{l}
\end{array}\right] .
\end{aligned}
$$

Mass, $M$, and stiffness, $C$, in particular are parameters that are highlighted as having a potentially significan influenc on the system. However, greater insight will be obtained through utilizing the expected variation of each parameter value as is used in control theory [15] by multiplying each value in the matrix by the ratio of expected variation divided by the nominal value for each parameter.

Furthermore, at this point let us conf ne the parameters considered to the design factors that can be investigated in physical experiments later, which relate to $R_{e}, M, R_{f t}$ and $R_{b f}$. Only the main matrix values modif ed by these design factors are shown below. In each case we have used the anticipated experimental range value as the expected variation value, that is we are calculating response sensitivity relative to the design factors.

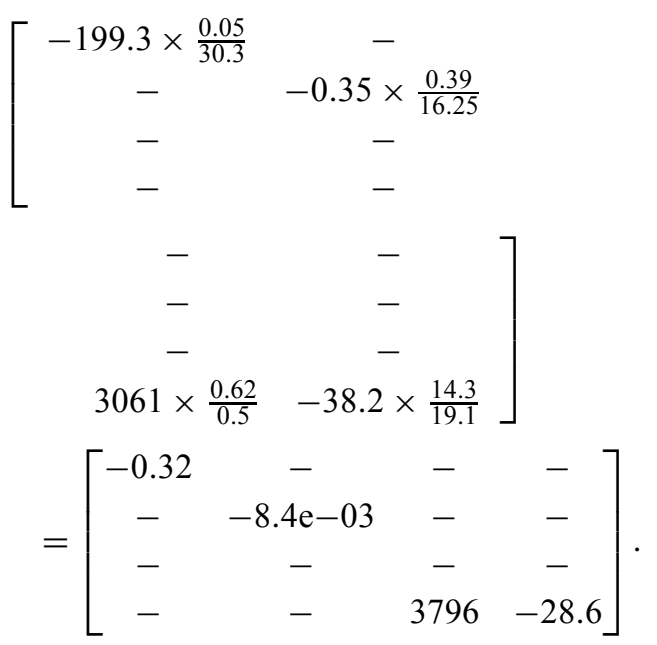

Note that from this analysis mass, $M$, is the strongest candidate followed by blade friction, $R_{b f}$. 
Table 4. Experimental results, blade speed measured using a stroboscope in flashe per second

\begin{tabular}{rcccccc}
\hline Run & $R_{e}(\Omega)$ & $R_{b f}\left(\mathrm{~N} \mathrm{~s} \mathrm{~m}^{-1}\right)$ & $R_{f t}\left(\mathrm{~N} \mathrm{~ms} \mathrm{r}^{-1}\right)$ & $M(\mathrm{~kg})$ & $S_{l}(\mathrm{~N})$ & Blade speed \\
\hline 1 & 30.30 & 19.1 & $15.86 \mathrm{e}-06$ & 0.5 & 25 & 2010 \\
2 & 30.30 & 6.3 & $16.06 \mathrm{e}-06$ & 0.81 & 25 & 1900 \\
3 & 30.30 & 4.8 & $16.25 \mathrm{e}-06$ & 1.12 & 25 & 1620 \\
4 & 30.26 & 19.1 & $16.06 \mathrm{e}-06$ & 1.12 & 25 & 1890 \\
5 & 30.26 & 6.3 & $16.25 \mathrm{e}-06$ & 0.5 & 25 & 1910 \\
6 & 30.26 & 4.8 & $15.86 \mathrm{e}-06$ & 0.81 & 25 & 1875 \\
7 & 30.25 & 19.1 & $16.25 \mathrm{e}-06$ & 0.81 & 25 & 1885 \\
8 & 30.25 & 6.3 & $15.86 \mathrm{e}-06$ & 1.12 & 25 & 1780 \\
9 & 30.25 & 4.8 & $16.06 \mathrm{e}-06$ & 0.5 & 25 & 1950 \\
10 & 30.30 & 19.1 & $15.86 \mathrm{e}-06$ & 0.5 & 45 & 1850 \\
11 & 30.30 & 6.3 & $16.06 \mathrm{e}-06$ & 0.81 & 45 & 1795 \\
12 & 30.30 & 4.8 & $16.25 \mathrm{e}-06$ & 1.12 & 45 & 1540 \\
13 & 30.26 & 19.1 & $16.06 \mathrm{e}-06$ & 1.12 & 45 & 1715 \\
14 & 30.26 & 6.3 & $16.25 \mathrm{e}-06$ & 0.5 & 45 & 1780 \\
15 & 30.26 & 4.8 & $15.86 \mathrm{e}-06$ & 0.81 & 45 & 1600 \\
16 & 30.25 & 19.1 & $16.25 \mathrm{e}-06$ & 0.81 & 45 & 1790 \\
17 & 30.25 & 6.3 & $15.86 \mathrm{e}-06$ & 1.12 & 45 & 1700 \\
18 & 30.25 & 4.8 & $16.06 \mathrm{e}-06$ & 0.5 & 45 & 1840 \\
\hline
\end{tabular}

Table 5. Linear regression results-most important factor in bold

\begin{tabular}{|c|c|c|c|c|c|c|c|c|}
\hline \multicolumn{6}{|c|}{ Regression model factors } & \multicolumn{3}{|c|}{ Regression statistics } \\
\hline Constant & $R_{e}$ & $R_{b f}$ & $R_{f t}$ & $M$ & $S_{l}$ & $R^{2}$-value & $F$-value & $p$-value \\
\hline 1811.0 & -42.917 & 93.288 & -49.543 & -188.39 & -137.13 & 0.81731 & 10.737 & $4.224 \mathrm{e}-04$ \\
\hline
\end{tabular}

\subsection{Physical experimentation}

Table 4 shows the experimental design plan (a repeated L9 orthogonal array) and results for the physical experimentation on the hedgetrimmer.

In this experiment $R_{e}, R_{b f}, R_{f t}$ and $M$ are considered to be design factors, $S_{l}$ is a noise factor and blade speed is the quality characteristic. A simple linear regression analysis of the experimental data is reported in Table 5. The factor values are scaled to be in the range $[-0.5,+0.5]$ so that direct comparisons may be made between model factors.

The model ftted to the data shows that $M$ is the most important design factor, followed by $R_{b f}$, as predicted by the bond graph model. Blade load, $S_{l}$, is also very important, but is outside the designer's control, so a good design would aim to reduce the strength of this effect. The regression statistics show that, with an $R^{2}$ value of $0.82,82 \%$ of the variability in the data are explained by the model, and the $F$ statistic of 10.7 and its $95 \% p$-value of 0.0004 show that the model parameters are highly unlikely to be zero.

\section{DISCUSSION}

Both case studies show a good agreement between the expected importance of the design factors, and the actual importance as measured using empirical data.

The loudspeaker case study involved collecting data on a single design and measuring the unit-to-unit variability introduced during the manufacture of the drive units. The data collected here showed only a small variation in product performance, but important effects were still discernable when the data were analysed. Several linear regression models were built at different frequency points, and the most reliable models (those built at $150-400 \mathrm{~Hz}$ ) all showed that the factor $R_{e}$ was the most important in determining the SPL response. This is consistent with the bond graph analysis, which also showed $R_{e}$ as being an important factor. The bond graph analysis also showed $B l$ as being important, and this factor was signif cant in the regression models ftted, but it was not as strong an influenc as $R_{e}$.

The hedgetrimmer study involved performing an L9 orthogonal array experiment on the hedgetrim- 
mer design. Performing an experiment on the design should lead to larger variation in design performance and show the relationships between design factors and response more clearly than measuring manufacturing variability. Indeed, this is what the results show with a statistically more significa $t$ linear regression model than those obtained for the loudspeaker study. The $\mathrm{ft}$ ted linear regression model explained $82 \%$ of the variation in blade speed. Again the f ndings from the bond graph analysis were conf rmed and showed that $M$ was the most important design factor, followed by $R_{b f}$.

\subsection{Conclusions}

The use of bond graphs in engineering design has been described and applied to two design studies. Some very encouraging preliminary results have been presented that show how bond graphs can be used to help in encapsulating engineering knowledge about a system and in selecting important design factors. This is useful in RED, where experiments are conducted on engineering systems by varying the settings of design factors.

The two studies involved analysis of empirical data collected during manufacture of a loudspeaker driver unit, and during a more general experiment on the design of a hedgetrimmer. These studies showed how the methods discussed might be applied in future to provide a more causal link between engineering design and performing experiments for system optimization using RED.

The work on bond graphs has been extended to analyse causal relationships between design factors, and tracing causal links to highlight potential interactions is proposed. Future work will establish closer links between bond graph causality and experimental design and model selection in RED for both real and computer experiments.

\section{ACKNOWLEDGEMENTS}

The authors gratefully acknowledge the technical contributions made by staff at the Control Laboratory of the University of Twente, Celestion (UK) and Black \& Decker.

\section{REFERENCES}

1. Paynter HM. Analysis and Design of Engineering Systems. MIT Press: Cambridge, MA, 1961.
2. Karnopp DC. Computer simulation of stick-slip friction in mechanical dynamic systems. Transcepts of ASME Journal of Dynamic Systems, Measurement, and Control, 1985; 107(1): $100-103$.

3. Rosenberg RC. Exploiting bond graph causality in physical systems models. Transcepts of ASME Journal of Dynamic Systems, Measurement, and Control, 1987; 109(4): 378-383.

4. Cellier FE. Hierarchical nonlinear bond graphs: a unif ed methodolgy for modelling complex physical systems. Proceedings of European Simulation Multiconference 1990 on Modelling and Simulation, B Schmidt (ed.). 1990; 1-13.

5. Karnopp DC. Energetically consistent bond graph models in electromechanical energy conversion. Journal of the Franklin Institute, 1990; 327(5): 677-686.

6. Rosenberg RC, Karnopp DC. Introduction to Physical System Dynamics. McGraw-Hill, 1983; 11-20.

7. Karnopp DC, Margolis DL, Rosenberg RC. System Dynamics-A Unifie Approach (2nd edn.). John Wiley and Sons, 1990; 14-400.

8. Rosenberg RC, Zhou, T. Power-based model insight. ASME 1988 Winter Annual Meeting, 1988DSC-8:61-68.

9. Taguchi G. System of Experimental Design, vols. 1 and 2. UNIPUB, 1987.

10. Phadke MS. Quality Engineering Using Robust Design. Prentice Hall, 1989, 27-30, 135.

11. Carter ADS. Mechanical Reliability and Design. Macmillan, 1997.

12. Martens HR, Bell AC. A logical procedure for the construction of bond graphs in systems modelling. Trancepts of ASME Journal of Dynamic Systems, Measurement, and Control, 1972; 94(3): 183-188.

13. Breedveld PC, Rosenberg RC, Zhou T. Bibliography of bond graph theory and application. Journal of Franklin Institute, 1991; 328(5/6): 1067-1109.

14. Colloms M. High Performance Loudspeakers (4th edn.). Pentech Press, 1991; 76-80.

15. Skogestad S, Postlethwaite I. Multivariable Feedback Control. John Wiley and Sons, 1996.

\section{Authors' biographies:}

Mark Atherton is a principal lecturer in the School of Engineering Systems \& Design at South Bank University, prior to which he worked for Rubery Owen, GEC, Otis Elevator and Redland Engineering. He gained a BSc(Hons) in mechanical engineering from Aston University, an MSc in Industrial Robotics \& Manufacturing Automation from Imperial College and a $\mathrm{PhD}$ in mechanical engineering design from City University, London. His research interests relate to engineering design for quality.

Ron Bates is a Senior Research Fellow in the Department of Statistics at the University of Warwick, and Director of Morphism Limited, a software and consultancy company which specializes in industrial experimental design, spatial modelling and numerical optimization methods. He has a BSc degree in Engineering from Sussex University and a $\mathrm{PhD}$ degree in Robust Engineering Design from City University, London. His research interests include design of experiments, modelling complex nonlinear engineering and biomedical systems, optimization and risk assessment methodology. 